\title{
Green Tea Polyphenol Epigallocatechin-3-O-Gallate Attenuates Lipopolysaccharide-induced Nitric Oxide Production in RAW264.7 Cells
}

\author{
Seung-Jae Lee ${ }^{1}$, Hyun Woo Kang ${ }^{2}$, Seung Yuan Lee ${ }^{1}$, Sun Jin Hur ${ }^{1, *}$ \\ ${ }^{1}$ Department of Animal Science and Technology, Chung-Ang University, Anseong, Korea \\ ${ }^{2}$ Department of Korean Food \& Culinary Arts, Youngsan University, Busan, Korea \\ *Corresponding author: hursj@cau.ac.kr
}

Received July 08, 2014; Revised July 18, 2014; Accepted July 24, 2014

\begin{abstract}
Epigallocatechin-3-O-gallate (EGCG), the major polyphenol found in green tea, has been shown to downregulate inflammatory responses in macrophages; however, the underlying mechanism has not been understood. Overproduction of nitric oxide (NO) by inducible nitric oxide synthase (iNOS) is known to be closely correlated with the pathology of a variety of diseases and inflammations. In this study, we investigated the inhibitory effect of EGCG on NO production and its molecular mechanism in lipopolysaccharide (LPS)-stimulated macrophage RAW264.7 cells. Besides a decrease in NO secretion, protein levels of inducible nitric oxide synthase (iNOS) and cyclooxygenase-2 (COX-2) also decreased in LPS-stimulated RAW264.7 macrophage cells treated with EGCG. These results suggest that EGCG possesses a potent anti-inflammatory activity.
\end{abstract}

Keywords: Epigallocatechin-3-O-gallate, Nitric oxide, Inflammation, RAW264.7 cells, Lipopolysaccharide

Cite This Article: Seung-Jae Lee, Hyun Woo Kang, Seung Yuan Lee, and Sun Jin Hur, "Green Tea Polyphenol Epigallocatechin-3-O-Gallate Attenuates Lipopolysaccharide-induced Nitric Oxide Production in RAW264.7 Cells." Journal of Food and Nutrition Research, vol. 2, no. 7 (2014): 425-427. doi: 10.12691/jfnr-27-16.

\section{Introduction}

Nitric oxide (NO) is a pleiotropic biological molecule involved in a myriad of physiological and pathological processes such as regulation of blood pressure, neurotransmission, signal transduction, anti-microbial defense [1,2], immunomodulation [3], cellular redox regulation [4,5], and apoptosis [6]. It is synthesized endogenously by nitric oxide synthases (NOSs) through the conversion of L-arginine to $\mathrm{NO}$ and L-citrulline $[7,8]$. Three types of NOS isoforms have been identified to date: neuronal NOS (nNOS), endothelial NOS (eNOS), and inducible NOS (iNOS) [9]. Among these, iNOS is highly expressed in lipopolysaccharide (LPS)-activated macrophages and the iNOS-derived NO plays a crucial role in macrophages-mediated inflammatory responses. However, overproduction of NO in certain conditions can lead to undesirable inflammatory reactions such as septic shock and tissue injuries $[10,11]$.

Green tea is one of the most widely consumed beverages in the world and its probable health benefits have been the subject of considerable attention [12]. The polyphenol EGCG, found abundantly in green tea, has been shown to possess a variety of biological and pharmacological properties including cancer-preventive, antiallergic, antioxidative, and anti-inflammatory activities $[13,14,15]$. In addition, EGCG is relatively safe [16], and green tea extract containing 60\% EGCG has been approved by the US Food and Drug Administration as the first botanical drug [17].

In this study, we investigated the effect of the green tea polyphenol EGCG on NO production in LPS-stimulated RAW264.7 macrophages and attempted to elucidate the underlying molecular mechanism.

\section{Materials and Methods}

\subsection{Materials}

Epigallocatechin-3-O-gallate, 3-[4,5-dimethy-thiazol-2yl]-2,5-diphenyl tetrazolium bromide (MTT) assay kit, and LPS from Escherichia coli (serotype 0127:B8) were procured from Sigma Aldrich (St. Louis, MO, USA). Tissue culture plates and culture dishes were purchased from Nunc, Inc. (North Aurora Road, IL, USA). The antibodies against COX-2, iNOS, and $\beta$-actin were supplied by Cell Signaling (Danvers, MA, USA). Dulbecco's modified Eagle's medium (DMEM), fetal bovine serum (FBS), penicillin, and streptomycin were purchased from Hyclone (Logan, UT, USA). Reagents for ECL Western blot detection were purchased from GE Healthcare Biosciences (Piscataway, NJ). All other reagents were of the highest grade available commercially.

\subsection{Cell Culture}

Mouse macrophage cell line, RAW264.7, was obtained from American Type Culture Collection (ATCC, 
Rockville, MD, USA) and cultured in DMEM supplemented with penicillin, streptomycin, and $10 \%$ heat-inactivated FBS in 5\% $\mathrm{CO}_{2}$ and $95 \%$ humidified air at $37^{\circ} \mathrm{C}$.

\subsection{MTT assay}

The cells were pro cells were plated at a density of $5 \times$ $10^{5}$ cells/well in a 96-well plate and treated with EGCG at $0.1,1.0$, or $10.0 \mu \mathrm{M}$ with or without LPS $50 \mathrm{ng} / \mathrm{mL}$, for a day prior to MTT assay. Following exposure, the cells were processed for the assay as per manufacturer's instructions. Briefly, the cells were incubated with the MTT solution for $4 \mathrm{~h}$ at $37^{\circ} \mathrm{C}$ in $5 \% \mathrm{CO}_{2}$. After incubation, the supernatant was removed and the cells were washed twice with PBS. The formazan crystals produced in viable cells were solubilized in $200 \mu \mathrm{L}$ of DMSO and absorbance was measured at $550 \mathrm{~nm}$ using a microplate reader (Tecan Trading AG, Männedorf, Switzerland). Experiments were performed in three replicates [13].

\subsection{NO Assay}

Production of NO was assayed using the Griess reaction [14] to measure the levels of nitrite, a stable NO metabolite, secreted in the culture medium. The RAW264.7 cells were plated in a 96-well plate at a density of $5 \times 10^{4}$ cells/well in $100 \mu \mathrm{L}$ of culture medium. Following attachment, cells were pretreated for $30 \mathrm{~min}$ with predetermined concentration of EGCG $(1.0 \mu \mathrm{M})$ and then stimulated with LPS, $50 \mathrm{ng} / \mathrm{mL}$, for $24 \mathrm{~h}$. Following LPS treatment, $50 \mu \mathrm{L}$ of supernatant from each well was transferred to a corresponding well in another plate containing $50 \mu \mathrm{L}$ of Griess reagent and incubated for 10 min at room temperature and absorbance measured at 540 nm using a microplate reader (Tecan Trading AG). Experiments were performed in triplicate.

\subsection{Western Blot}

Cell lysates were prepared according to a previously described methodology [15] and protein concentration was determined using Bradford assay (Bio-Rad; Hercules, CA, USA). $50 \mathrm{mg}$ of protein in each sample was resolved using 7.5\% SDS-polyacrylamide gel electrophoresis (SDS-PAGE), transferred to a PVDF membrane, and incubated with the appropriate antibodies. Following secondary antibody application, the, membrane was washed three times with TBS-T and developed for visualization on a Luminescent image analyzer (LAS3000, Fujifilm, Tokyo, Japan) by using an ECL detection kit.

\subsection{Statistical Analysis}

Study data are expressed as mean \pm standard error of mean (SEM). Statistical analyses of differences between treatment groups were conducted using Student's $t$-test for paired data, and $p<0.05$ was considered to have statistical significance. All analyses were carried out in triplicate using Graph Pad Prism software version 4.00 (Graph Pad Software Inc., San Diego, CA).

\section{Results}
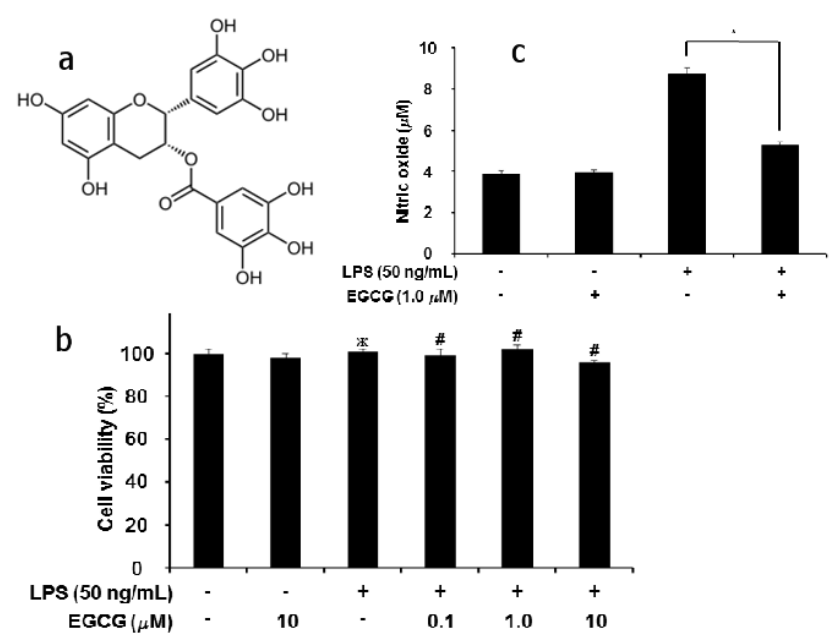

Figure 1. Effect of EGCG on cell viability and induced NO production in RAW264.7 cells, with or without LPS-stimulation. (A) Chemical structure of EGCG. (B) Cell viability of RAW264.7 cells incubated with $0.1,1.0$, and $10 \mu \mathrm{M}$ EGCG and LPS $(50 \mathrm{ng} / \mathrm{mL})$ for $24 \mathrm{~h}$ determined using MTT assay. (C) NO concentration in the culture medium following treatment with EGCG $(1.0 \mu \mathrm{g} / \mathrm{mL})$ and/or LPS $(50 \mathrm{ng} / \mathrm{mL})$ determined using Griess assay. Data are presented as mean \pm S.E.M. $(n=3)$ for three independent experiments. жNot significantly different from that of the control group. \#Not significantly different from the LPS-treated group. Significance was determined by Student's $t$-test. ${ }^{*} p<0.05$
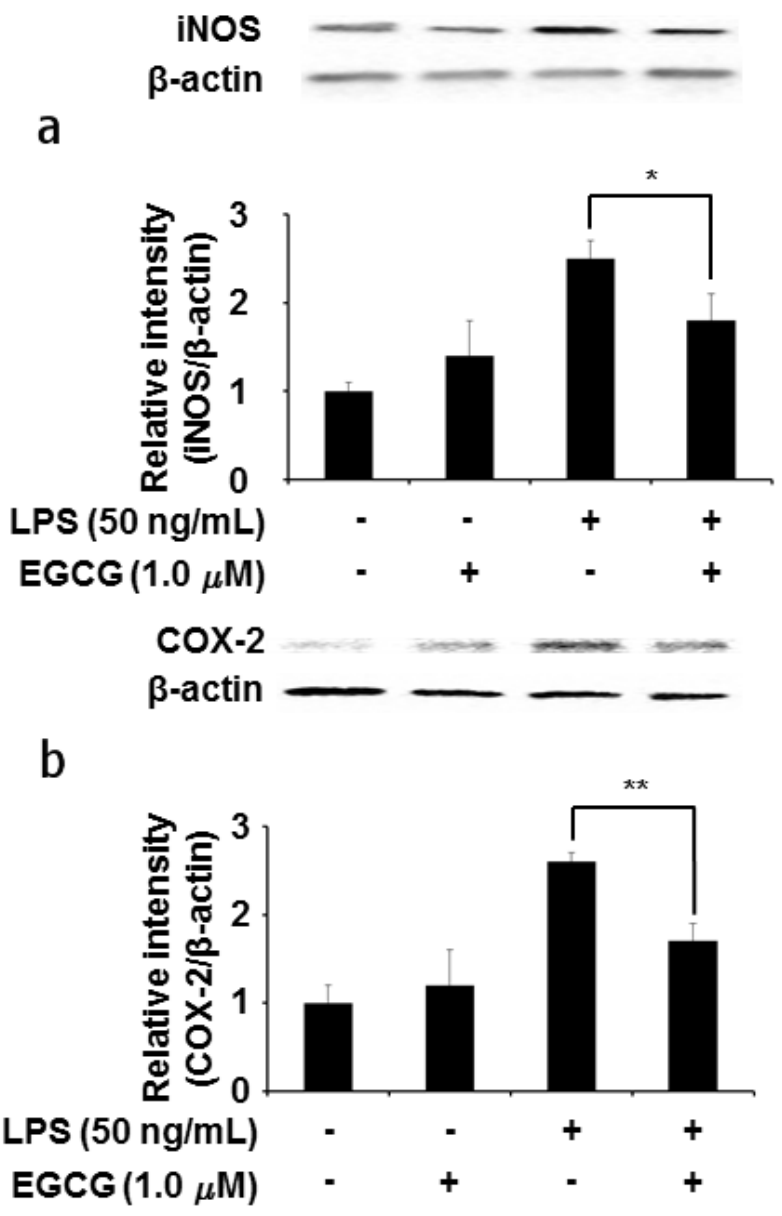

Figure 2. Effect of EGCG on LPS-induced iNOS (A) and COX-2 (B) expression. Relative expression of iNOS and COX-2 to control ( $\beta$-actin) was performed by densitometric analysis of gels (lower). ${ }^{*} p<0.05$ and ${ }^{* *} p<0.01$ are significantly different as analyzed by paired $t$-test compared with LPS-stimulated group

The effect of EGCG at concentrations of 0.1, 1.0, and $10 \mu \mathrm{M}$ on the viability of RAW264.7 cells following 
exposure for $24 \mathrm{~h}$ was examined by performing the MTT assay. As shown in Figure 1B, cell viability was not reduced by EGCG up to $10 \mu \mathrm{M}$ even when the cells were concomitantly treated with LPS at $50 \mathrm{ng} / \mathrm{mL}$. Based on these results, an EGCG concentration of $1.0 \mu \mathrm{M}$ was selected for further experiments. To investigate whether EGCG of green tea could inhibit LPS-induced NO production, RAW264.7 cells were pretreated for $4 \mathrm{~h}$ with 1.0 $\mu \mathrm{M}$ EGCG followed by stimulation with LPS at 50 $\mathrm{ng} / \mathrm{mL}$ for $18 \mathrm{~h}$. The level of NO in the culture media was determined using the Griess reagent. Cells treated with LPS alone showed a notable increase in NO production compared to negative control and this upsurge was substantially attenuated in cells that were pretreated with EGCG, $1.0 \mu \mathrm{M}$ (Figure 1C). NO plays a central role in the physiology and pathology of diverse organs including the immune system. Further investigations were carried out to ascertain whether decreased expression of iNOS and COX-2 at the protein level correlated with the EGCGmediated suppression of NO production. Western blot analysis (Figure 2) of RAW264.7 cells treated as in the NO assay revealed that iNOS and COX-2 protein levels increased following LPS-stimulation. Moreover, this increase in protein expression was considerably mitigated in the cells pretreated with $1.0 \mu \mathrm{g} / \mathrm{ml} \mathrm{EGCG} \mathrm{(Figure} \mathrm{2).}$ These results correlate well with the EGCG-mediated inhibition of NO production in activated macrophages, suggesting that suppression of iNOS and COX-2 at the protein level plays a major role in the anti-inflammatory mechanism of EGCG.

\section{Discussion}

Proinflammatory cytokines work in a synergistic manner through a cytokine transcription factor regulatory loop, thereby augmenting the inflammatory response and tissue damage [21]. NO is an important inflammatory mediator produced by NOS under physiological and pathophysiological conditions and downregulation of NO is necessary for the treatment of the latter [19]. The production of NO and prostaglandins by iNOS and COX2 , respectively, is considered to be the most prominent molecular mechanism in inflammatory processes [22,23], and is also involved in multistage carcinogenesis, especially at the promotion stage [24]. Excessive and prolonged NO generation caused by overexpression of iNOS has been implicated in inflammational tumorigenesis, while COX-2-mediated prostaglandin production has been shown to stimulate cell proliferation, invasion, and angiogenesis in cancer development [25]. In the past, EGCG has proven to be an effective drug in the treatment of several diseases such as cardiovascular disease, cancer, and neurodegeneration owing to its strong antioxidant and anti-inflammatory effects [26,27,28,29]. Previous studies have also shown that EGCG improves glucose tolerance, protects pancreatic islets, ameliorates aortic reactivity and the development of diabetic nephropathy [30], suppresses high glucose-induced apoptosis, ameliorates hyperglycemia-induced embryonic vasculopathy, and attenuates glucotoxicity [31]. Moreover structural modification of EGCG to produce lipophilic ester derivatives has been shown to enhance its cellular absorption and bioefficiency in vivo [32], thereby indicating the possibility of improvement in its pharmacokinetic profile.

In the present study, we showed that EGCG inhibited the production of NO, iNOS, and COX-2 in LPSstimulated RAW264.7 cells. However, iNOS, COX-2, and pro-inflammatory cytokine expression may be regulated through different pathways in immunoregulatory signaling. Thus, further whole-animal studies are warranted to elucidate the molecular mechanisms underlying the antiinflammatory properties of EGCG.

\section{Conclusions}

The current study demonstrated that green tea polyphenol EGCG has an ameliorative effect on the LPSinduced iNOS expression and NO production in activated macrophages. Natural products have been historically used in traditional medicine and are now proving to be a potential source of new drugs and nutraceuticals. In light of this, our study verifying the immunomodulatory activity of EGCG and the possible underlying mechanism would contribute to the understanding of the biological properties of green tea and its further consideration for therapeutic applications.

\section{Acknowledgments}

This research was supported by Youngsan University in 2014.

\section{References}

[1] MacMicking, J., Xie, Q.W., and Nathan, C., "Nitric oxide and macrophage function”, Annu Rev Immunol 15, 323-350. 1997.

[2] Schoedon, G., Schneemann, M., Walter, R., Blau, N., Hofer, S., and Schaffner, A., "Nitric oxide and infection: another view" Clin Infect Dis 21(Suppl 2), S152-157. 1995.

[3] Gomez-Flores, R., Weber, R.J., "Immunomodulation of macrophage functions by opioids” Adv Exp Med Biol 437, 13-19. 1998.

[4] Forman, H.J., and Torres, M., "Redox signaling in macrophages" Mol Aspects Med 22 (4-5), 189-216. 2001.

[5] Kim, S., and Ponka, P., "Nitric oxide-mediated modulation of iron regulatory proteins: implication for cellular iron homeostasis" Blood Cells Mol Diseases 29 (3), 400-410. 2002.

[6] Yang, C.S., Wang, X., Lu, G., and Picinich, S.C., "Cancer prevention by tea: animal studies, molecular mechanisms and human relevance” Nat Rev Cancer 9 (6), 429-439. 2009.

[7] Tsai, S.Y., Chang, Y., Chen, TL., and Chen, R.M., "Therapeutic concentrations of propofol protects mouse macrophages from nitric oxide-induced cell death and apoptosis” Can J Anaesth 49 (5), 477-480. 2002.

[8] Hobbs, A.J., Higgs, A., and Moncada, S., "Inhibition of nitric oxide synthase as a potential therapeutic target” Annu Rev Pharmacol Toxicol 39, 191-220. 1999.

[9] Marletta, M.A., "Nitric oxide synthase structure and mechanism" J Biol Chem 268 (17), 12231-12234. 1993.

[10] Chung, H.T., Pae, H.O., Choi, B.M., Billiar, T.R., and Kim, Y.M., "Nitric oxide as a bioregulator of apoptosis, Biochem" Biophys Res Commun 282 (5), 1075-1079. 2001.

[11] Xie, Q.W., Cho, H.J., Calaycay, J., Mumford, R.A., Swiderek, K.M., and Lee, T.D., "Cloning and characterization of inducible nitric oxide synthase from mouse macrophages" Science 256 (5054), 225-228. 1992.

[12] Yang, C.S., Maliakal, P., and Meng, X., "Inhibition of carcinogenesis by tea” Annu Rev Pharmacol Toxicol 42, 25-54. 2002. 
[13] Lin, Y.L., and Lin, J.K., “(-)-Epigallocatechin-3-gallate blocks the induction of nitric oxide synthase by down-regulating lipopolysaccharide-induced activity of transcription factor nuclear factor-kappaB” Mol Pharmacol 52 (3), 465-472. 1997.

[14] Bors, W., and Saran, M., "Radical scavenging by flavonoid antioxidants” Free Radic Res Commun 2 (4-6), 289-294. 1987.

[15] Fujimura, Y., Tachibana, H., and Yamada, K., “A tea catechin suppresses the expression of the high-affinity IgE receptor Fc $\varepsilon$ RI in human basophilic KU812 cells” J Agric Food Chem 49(5), 2527-2531. 2001.

[16] Chow, H.H., Cai, Y., Hakim, I.A., Crowell, J.A., Shahi, F., Brooks, C.A., Dorr, R.T., Hara, Y., and Alberts, D.S., "Pharmacokinetics and safety of green tea polyphenols after multiple-dose administration of epigallocatechin gallate and polyphenon $\mathrm{E}$ in healthy individuals” Clin Cancer Res 9 (9), 3312-3319. 2003.

[17] Wu, K.M., Ghantous, H., and Birnkrant, D.B., "Current regulatory toxicology perspectives on the development of herbal medicines to prescription drug products in United States” Food Chem Toxicol 46 (8), 2606-2610. 2008.

[18] Lee, S.J., Kim, E.K., Kim, Y.S., Hwang, J.W., Lee, K.H., Choi, D.K., Kang, H., Moon, S.H., Jeon, B.T., and Park, P.J., "Purification and characterization of a nitric oxide inhibitory peptide from Ruditapes philippinarum” Food Chem Toxicol 50 (5), 1660-1666. 2012.

[19] Ma, J.S., Kim, W.J., Kim, J.J., Kim, T.J., Ye, S.K., Song, M.D., Kang, H., Kim, D.W., Moon, W.K., and Lee, K.H., "Gold nanoparticles attenuate LPS-induced NO production through the inhibition of NF-jB and IFN-b/STAT1 pathways in RAW264.7 cells” Nitric Oxide 23 (3), 214-219. 2010.

[20] Lee, S.J., Kim, Y.S., Hwang, J.W., Kim, E.K., Moon, S,H., Jeon, B.T., Jeon, Y.J., Kim, J.M., and Park, P.J., "Purification and characterization of a novel antioxidative peptide from duck skin by-products that protects liver against oxidative damage" Food Res Int 49 (1), 285-295. 2012.
[21] Fiocchi, C., "Inflammatory bowel disease: Etiology and pathogenesis” Gastroenterology 115 (1), 182-205. 1998

[22] Moncada, S., "Nitric oxide: Discovery and impact on clinical medicine” J Roy Soc Med 92 (4), 164-169. 1999.

[23] Turini, M.E., and DuBois, R.N., "Cyclooxygenase-2: A therapeutic target” Annu Rev Med 53, 35-57. 2002.

[24] Pan, M.H., and Ho, C.T., "Chemopreventive effects of natural dietary compounds on cancer development” Chem Soc Rev 37 (11) 2558-2574. 2008

[25] Mann, J.R., Backlund, M.G., and DuBois, R.N., "Mechanisms of disease: Inflammatory mediators and cancer prevention” Nat Clin Pract Oncol 2 (4), 202-210. 2005.

[26] Grelle, G., Otto, A., Lorenz, M., Frank, R.F., Wanker, E.E., and Bieschke, J., "Black tea theaflavins inhibit formation of toxic amyloid-beta and alpha-synuclein fibrils” Biochemistry 50 (49), 10624-10636. 2011.

[27] Wolfram, S., "Effects of green tea and EGCG on cardiovascular and metabolic health” J Am Coll Nutr 26 (4), 373S-88S. 2007.

[28] Ghosh, S., May, M.J., and Kopp, E.B., “NF-kappa B and Rel proteins: evolutionarily conserved mediators of immune responses” Annu Rev Immunol 16, 225-260. 1998.

[29] Liu, R.H., and Hotchkiss, J.H., "Potential genotoxicity of chronically elevated nitric oxide: a review” Mutat Res 339 (2), 7389. 1995.

[30] Roghani, M., and Baluchnejadmojarad, T., "Chronic epigallocatechin-gallate improves aortic reactivity of diabetic rats: underlying mechanisms" Vascul Pharmacol 51 ( ), 84-9. 2009.

[31] Yang, P., and Li, H., "Epigallocatechin-3-gallate ameliorates hyperglycemia-induced embryonic vasculopathy and malformation by inhibition of Foxo3a activation” Am J Obstet Gynecol 203 (75), 1-6. 2010.

[32] Zhong, Y., Chiou, Y.S., Pan, M.H., and Shahidi, F., "Antiinflammatory activity of lipophilic epigallocatechin gallate (EGCG) derivatives in LPS-stimulated murine macrophages" Food Chem 134 (), 742-748. 